\title{
Natural history of large regenerative nodules and dysplastic nodules in liver cirrhosis: 28-year follow-up study
}

\author{
Tsunenobu Sato $\cdot$ Fukuo Kondo $\cdot$ Masaaki Ebara $\cdot$ Nobuyuki Sugiura $\cdot$ \\ Shinichiro Okabe $\cdot$ Masahiko Sunaga $\cdot$ Masaharu Yoshikawa Eiichiro Suzuki $\cdot$ \\ Sadayuki Ogasawara Yusuke Shinozaki Yoshihiko Ooka · Tetsuhiro Chiba • \\ Fumihiko Kanai - Takashi Kishimoto · Yukio Nakatani - Toshio Fukusato • \\ Osamu Yokosuka
}

Received: 18 October 2014/Accepted: 31 January 2015/Published online: 3 March 2015

(C) The Author(s) 2015. This article is published with open access at Springerlink.com

\begin{abstract}
Background and aims Some follow-up studies of large regenerative nodules (LRNs) and dysplastic nodules (DNs) were reported previously. However, the pre-malignant potentiality of LRNs has remained controversial up to now. No LRNs showed malignant transformation in our previous study. We aimed to evaluate the pre-malignant potentiality of LRNs and DNs with a greater number of cases and longer follow-up periods.

Methods From 1982 to 2005, 1,500 consecutive nodular lesions up to $2 \mathrm{~cm}$ in diameter were subjected to US guided thin-needle biopsy in cirrhotic patients at Chiba University Hospital. Of these lesions, 68 LRNs in 60 cases and 20 DNs in 22 cases were followed up for more than 6 months without any anti-cancer therapy. The last US examination was in 2010 . The total study period was 28 years. We
\end{abstract}

T. Sato · M. Yoshikawa · E. Suzuki - S. Ogasawara · Y. Ooka ·

T. Chiba · F. Kanai · O. Yokosuka

Department of Gastroenterology and Nephrology, Graduate

School of Medicine, Chiba University, 1-8-1 Inohana, Chuo-ku,

Chiba City, Chiba 260-8670, Japan

e-mail: tsune-sato@cnc.jp; ccmc.tsato@gmail.com

M. Yoshikawa

e-mail: yoshikawa@faculty.chiba-u.jp

E. Suzuki

e-mail: eiichiro0709@ hotmail.com

S. Ogasawara

e-mail: sadahisa@me.com

Y. Ooka

e-mail: ooka-y@umin.ac.jp

T. Chiba

e-mail: techiba@faculty.chiba-u.jp

F. Kanai

e-mail: kanaif@faculty.chiba-u.jp analyzed the histological findings and the clinical data of all cases retrospectively. The outcome of the lesions was examined.

Results The mean follow-up period was 38.9 (16-119) months in LRNs and 31.9 (6-101 months) in DNs. Rate of nodule enlargement was higher in DNs ( $8 / 24$ nodules, $33 \%)$ than LRNs (11/68 nodules, $16 \%),(p=0.0743$, not significant). Rate of malignant transformation was also higher in DNs (10/24 nodules, $42 \%)$ than LRNs $(9 / 68$ nodules, $13 \%),(p=0.0040$, significant $)$. The rate of disappearance in images was similar between LRNs and DNs.

Conclusions We should recognize LRN as low risk premalignant lesions whereas DNs as high risk lesions.

Keywords Large regenerative nodule - Dysplastic nodule $\cdot$ Natural history $\cdot$ Malignant transformation

O. Yokosuka

e-mail: yokosukao@faculty.chiba-u.jp

T. Sato $\cdot$ M. Sunaga

Department of Gastroenterology, Chiba Central Medical Center, 1835-1 Kasoricho, Wakaba-ku, Chiba City, Chiba 264-0017,

Japan

e-mail: sunaga-m@umin.ac.jp

F. Kondo $(\square)$

Department of Pathology, Teikyo University Hospital, 2-11-1

Kaga, Itabashi-ku, Tokyo 173-8606, Japan

e-mail: fkondo55@med.teikyo-u.ac.jp

M. Ebara $\cdot$ S. Okabe

Department of Gastroenterology, Matsudo City Hospital, 4005

Kamihongo, Matsudo City, Chiba 271-8511, Japan

e-mail: masaaki-ebara@nifty.com

S. Okabe

e-mail: okabeshin1966@yahoo.co.jp 


$\begin{array}{ll}\text { Abbreviations } \\ \text { LRN } & \text { Large regenerative nodule } \\ \text { DN } & \text { Dysplastic nodules } \\ \text { HCC } & \text { Hepatocellular carcinoma } \\ \text { US } & \text { Ultrasound } \\ \text { CT } & \text { Computed tomography } \\ \text { MRI } & \text { Magnetic resonance image } \\ \text { HCV } & \text { Hepatitis C virus } \\ \text { HBV } & \text { Hepatitis B virus }\end{array}$

\section{Introduction}

Although several follow-up studies of large regenerative nodules (LRNs) and dysplastic nodules (DNs) have been reported [1-9], the pre-malignant potentiality of LRNs is still controversial. No LRNs showed malignant transformation in our previous study [1], whereas some authors reported that LRNs were pre-malignant $[3,8,9]$. However, these earlier reports are not easily compared, as the histological criteria of well differentiated hepatocellular carcinoma (HCC), DN and LRN were lacking universal consensus at that time $[10,11]$. In addition, the numbers of nodules were not so many and the follow-up periods were not so long. A few reports studied over 90 nodules [8,9], with the others studied less than 40 nodules [1-6]. In four of the nine previous studies, the longest follow-up period was less than 5 years. If the numbers of nodules and the lengths of the follow-up periods had been greater, the results might also have been different.

\footnotetext{
N. Sugiura $\cdot$ Y. Shinozaki

Department of Internal Medicine, National Hospital Organization Chiba Medical Center, 4-1-2 Tsubakimori, Chuo-ku, Chiba City, Chiba 260-8606, Japan

e-mail: sugiura@nho-chiba.hosp.go.jp

Y. Shinozaki

e-mail: y-shino@alpha.ocn.ne.jp

T. Kishimoto

Department of Molecular Pathology, Graduate School of Medicine, Chiba University, 1-8-1 Inohana, Chuo-ku, Chiba City, Chiba 260-8670, Japan

e-mail: tkishi@faculty.chiba-u.jp

Y. Nakatani

Department of Diagnostic Pathology, Graduate School of Medicine, Chiba University, 1-8-1 Inohana, Chuo-ku, Chiba City, Chiba 260-8670, Japan

e-mail: nakatani@faculty.chiba-u.jp

T. Fukusato

Department of Pathology, School of Medicine, Teikyo

University, 2-11-1 Kaga, Itabashi-ku, Tokyo 173-8605, Japan

e-mail: fukusato@med.teikyo-u.ac.jp
}

In the present study, we studied many more LRNs than in our previous study, and the follow-up period was also elongated. DNs were also studied and compared with the results of LRNs. This is the only study which dealt with more than 90 nodules smaller than $2 \mathrm{~cm}$ in which the longest follow-up period was more than 5 years.

\section{Materials and methods}

From 1982 to 2005,1500 consecutive nodular lesions up to $2 \mathrm{~cm}$ in diameter were subjected to ultrasound (US) guided thin-needle biopsy in cirrhotic patients at Chiba University Hospital. They were diagnosed as 898 HCCs, 108 DNs, 315 LRNs, and 179 other lesions. Of the 108 DNs, 76 DNs were treated according to the patient's preference or were confirmed as HCC by imaging diagnosis within 6 months. The remaining 32 DNs were followed up without any anti-cancer therapy. Informed consent of liver biopsy was taken from each patient. Within 6 months, eight DNs dropped out. Finally, 24 DNs from 22 cases were followed up for longer than 6 months. Similarly, 68 LRNs from 60 cases were followed up for more than 6 months. The last US examination of this study was performed in 2010, meaning that the total study period was 28 years (1982-2010). At this final point, we analyzed the histological findings and the clinical data of all cases retrospectively.

The outcome of the lesions (i.e., no change, enlargement of nodule size, malignant transformation and disappearance in images) was examined.

Profiles of the cases and nodules are shown in Tables 1 and 2, including age, male/female ratio, pre-existing HCC nodules, single or multiple lesions, diabetes mellitus (DM), liver function tests and tumor markers. No significant differences were seen between LRNs and DNs in respect to these items. As to the causes of cirrhosis in LRNs and DNs, hepatitis $\mathrm{C}$ virus was the most common, followed by hepatitis B virus. Cases of alcoholic cirrhosis were not included in this study (Table 1). Median nodule size was $12 \mathrm{~mm}$ in both LRNs and DNs (Table 2). Findings of ultrasound, dynamic CT and MRI were not statistically different between LRNs and DNs.

\section{Measurement of nodule size}

The longer diameter of the nodule shown on the monitor of the ultrasound (US) was measured. We used a pre-installed measuring tool in the US instrument. To avoid a measurement error, more than one doctor performed US examination. As a principle, one of them was a wellexperienced (more than 10 years) doctor. In case the longer 
Table 1 Profile of the cases

\begin{tabular}{llll}
\hline Characteristic & Large regenerative nodules (LRNs) & Dysplastic nodules (DNs) & $p$ value \\
\hline Nodule, $n$ & 68 & 24 & \\
Case, $n$ & 60 & 22 & $0.6080^{\mathrm{a}}$ \\
Age & $66(24-79)$ & $63(37-71)$ & $0.1815^{\mathrm{b}}$ \\
Male/female & $39 / 21$ & $18 / 4$ & $0.2730^{\mathrm{b}}$ \\
Pre-HCC $(+) /(-)$ & $10 / 44$ & $1 / 21$ & $1.0000^{\mathrm{b}}$ \\
Single nodule case, $n$ & 55 & 21 & \\
Multiple nodule case, $n$ & 5 & 1 & $0.8677^{\mathrm{c}}$ \\
Etiology & & & \\
HCV & 44 & 16 & \\
HBV & 8 & 4 & $1.0000^{\mathrm{b}}$ \\
HCV and HBV & 1 & 0 & $0.7529^{\mathrm{c}}$ \\
Alcohol & 0 & 0 & $0.6787^{\mathrm{a}}$ \\
Others & 7 & 2 & $0.5641^{\mathrm{a}}$ \\
DM $(+) /(-)$ & $7 / 53$ & $3 / 19$ & $0.2231^{\mathrm{a}}$ \\
Child classification A/B/C & $53 / 4 / 3$ & $19 / 1 / 2$ & $0.5040^{\mathrm{a}}$ \\
AST (IU/ml) & $72(15-400)$ & $57(34-197)$ & $0.1755^{\mathrm{a}}$ \\
ALT $(\mathrm{IU} / \mathrm{ml})$ & $69(11-466)$ & $63(27-307)$ & $12.1(5.5-21.0)$ \\
Platelet $\left(\times 10^{4} / \mu \mathrm{l}\right)$ & $11.0(3.7-20.9)$ & $10.5(3.7-55.3)$ & $15(<10-24)$ \\
AFP $(\mathrm{ng} / \mathrm{ml})$ & $8.2(1.8-657)$ & & \\
PIVKA-II $(\mathrm{mAU} / \mathrm{ml})$ & $18(<10-31)$ & &
\end{tabular}

Table 2 Profile of nodule

\begin{tabular}{llll}
\hline Parameter & Large regenerative nodules (LRNs) & Dysplastic nodules (DNs) & $p$ value \\
\hline Nodule, $n$ & 68 & 24 & \\
Median size (diameter, mm) & $12(8-20)$ & $12(8-17)$ & $0.8891^{\mathrm{a}}$ \\
US, hypo/hyper & $53 / 15$ & $14 / 10$ & $0.1071^{\mathrm{b}}$ \\
Dynamic CT & & & \\
Plain phase, high/iso/low & $1 / 58 / 6$ & $0 / 18 / 4$ & $0.4521^{\mathrm{c}}$ \\
Arterial phase, high/iso/low & $4 / 54 / 7$ & $0 / 19 / 5$ & $0.2434^{\mathrm{c}}$ \\
Late phase, high/iso/low & $2 / 52 / 11$ & $0 / 19 / 5$ & $0.6411^{\mathrm{c}}$ \\
MRI & & & \\
T1WI, high/iso/low & $7 / 33 / 2$ & $4 / 7 / 0$ & $0.2996^{\mathrm{c}}$ \\
T2WI, high/iso/low & $6 / 33 / 3$ & $0 / 10 / 1$ & $0.4109^{\mathrm{c}}$ \\
\hline
\end{tabular}

diameter increased 1.5 times the former size, we interpreted that the diameter increased significantly.

Definition of malignant transformation

Histological and/or imaging features which suggest HCC were used as the criteria of malignant transformation.

We evaluated nodule enlargement and malignant transformation independently. A nodule which showed enlargement of the size without malignant features in histological and imaging studies was simply interpreted as enlargement, whereas a nodule which showed malignant changes without enlargement was classified as malignant transformation.
Statistical analysis

In order to compare the various data of LRNs and DNs, the following statistical methods were used.

Mann-Whitney test: median age, median nodule size, AST, ALT, platelet, AFP, PIVKA-II

Fisher's exact test: male/female ratio, pre-existing HCC nodules, single or multiple lesions, US findings of nodules, diabetes mellitus

Chi-square test: etiology of cirrhosis, CT and MRI findings of nodules, occurrence of new HCC lesions remote from nodules, Child classification 
Log-rank (Mantel-Cox) test: rate of nodule enlargement, rate of progression to malignancy, rate of disappearance on ultrasonography

$p$ value less than 0.05 was recognized as significant.

\section{Results}

The mean follow-up period was 38.9 (16-119) months for LRNs and 31.9 (6-101 months) for DNs. Of the $68 \mathrm{LRNs}$, 11 showed enlargement and 35 became undetectable on US. Of the $24 \mathrm{DNs}$, eight showed enlargement and eight disappeared on US. All of the LRNs and DNs which disappeared remained undetectable by other types of imaging (CT and MRI) during the additional follow-up periods. As for the disappeared nodules, HCCs were not found in their loci. Finally, malignant transformation was proven in nine LRNs and ten DNs.

Comparison of outcomes of LRNs and DNs

\section{Rate of nodule enlargement}

Figure 1 shows the rates of nodule size enlargement in both LRNs and DNs. Using the Kaplan-Meier method, the rates of enlargement at 50 months and 100 months were 13.7 and $46 \%$ in LRNs and 26.5 and $69.4 \%$ in DNs, respectively. Nodule enlargement was more frequently found in DNs than in LRNs, although the difference was not significant $[p=0.0743$, Log-rank (Mantel-Cox) Test].

\section{Rate of progression to malignancy}

The rates of progression to malignancy in LRNs and DNs are shown in Fig. 2. The rate of LRNs to HCC was $13.6 \%$

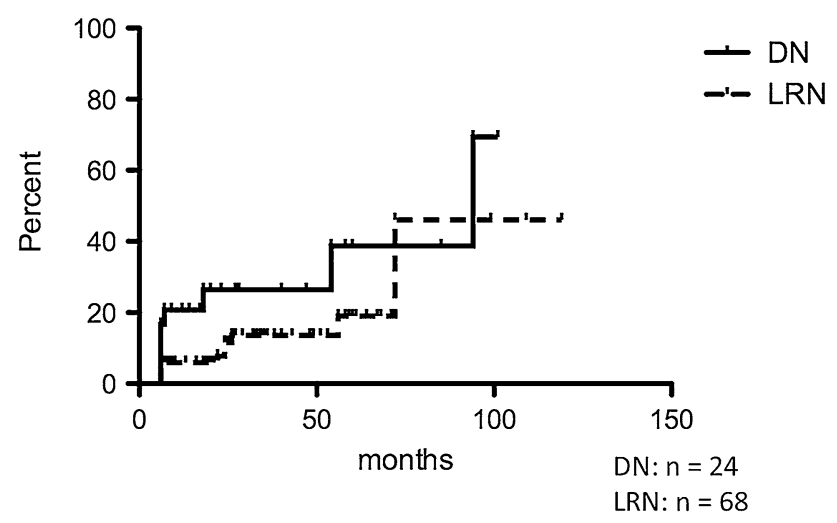

Fig. 1 Rate of nodule enlargement. Nodule enlargement was more frequently found in dysplastic nodules than in large regenerative nodules, although the difference was not significant $[p=0.0743$, Log-rank (Mantel-Cox) test]. DN dysplastic nodule, LRN large regenerative nodule at 50 months and $32 \%$ at 100 months, while that of DNs was $40 \%$ at 50 months and $75 \%$ at 100 months, using the Kaplan-Meier method. Progression to malignancy was more frequently found in DNs than in LRNs, with the difference being statistically significant $[p=0.0040$, Logrank (Mantel-Cox) Test].

Figure 3 shows a representative case of proven malignant transformation of LRN. The patient was a 60-year-old male, HCV antibody-positive, with liver cirrhosis. A hypoechoic nodule $13 \mathrm{~mm}$ in diameter was detected by US (Fig. 3a). Biopsy diagnosis was a large regenerative nodule (Fig. 3b). The nodule showed no change in size and character for 26 months (Fig. 3c), but at 32 months, the nodule had grown to $23 \mathrm{~mm}$ (Fig. 3d). Rebiopsy revealed its progression to well differentiated HCC (Fig. 3e). This LRN kept a benign nature for 26 months and then progressed to HCC within another 6-month period.

Comparisons of initial profiles including age, serum blood test and tumor markers in cases with and without malignant transformation of nodules are shown in Table 3. Among these items, only one item, i.e. DN/LRN, was proven to be related to malignant transformation ( $p=0.0066$, Fisher's exact test). Other items such as, nodule size, imaging findings, age, liver function tests, etiology of liver cirrhosis, tumor markers and association of DM were not related to malignant transformation.

\section{Disappearance on ultrasonography}

Figure 4 shows the rate of detection of LRNs and DNs by US. During the follow-up period, some LRNs and DNs became undetectable by US, and they were also not detectable by other imagings (CT, MRI). The detection rates of nodules at 50 and 100 months were 50.3 and $17.8 \%$ in

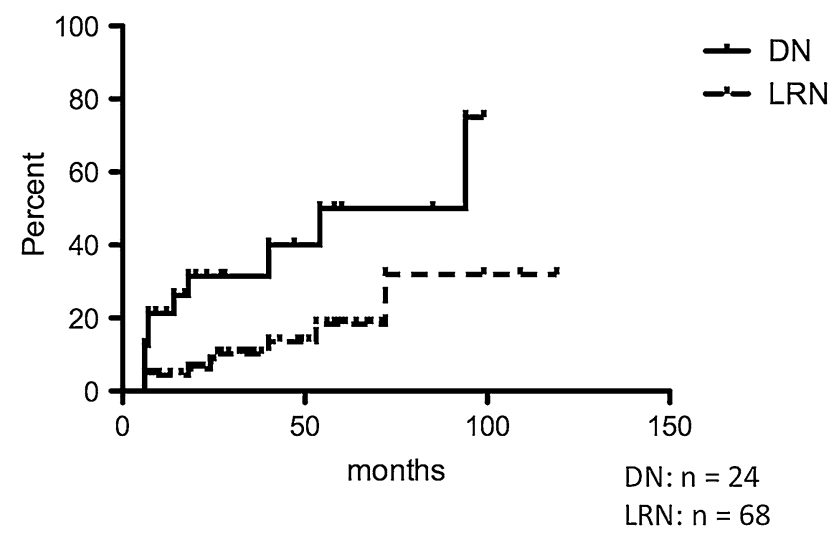

Fig. 2 Rate of progression to malignancy. Malignant transformation was more frequently found in dysplastic nodules than in large regenerative nodules. The difference was statistically significant $[p=0.0040$, Log-rank (Mantel-Cox) Test]. $D N$ dysplastic nodule, $L R N$ large regenerative nodule 

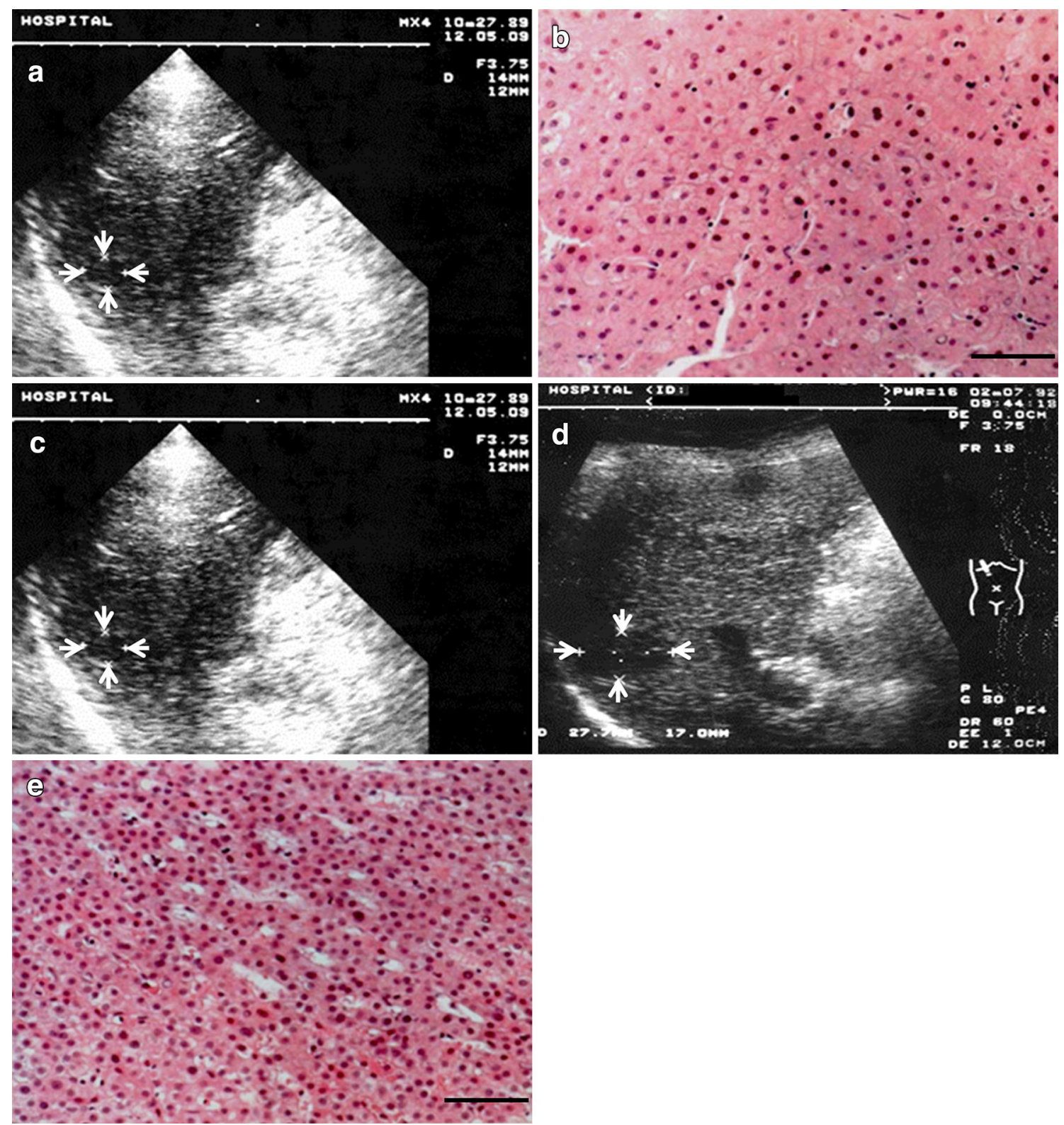

Fig. 3 An LRN case showing malignant transformation. a A hypoechoic nodule $13 \mathrm{~mm}$ in diameter was detected by US (arrows). b Biopsy specimen showed minimal atypia. Diagnosis was large regenerative nodule (bar $50 \mu \mathrm{m}$ ). c Size and US findings showed no

LRNs and 68.5 and $41.1 \%$ in DNs, according to the Kaplan-Meier method. There was no significant difference between LRNs and DNs. Various clinical data including DM, liver function tests and tumor markers shown in the Tables 1 and 3 were not proven to be related to the disappearance of the nodules.

\section{Occurrence of new HCC lesions remote from nodules}

Table 4 shows the frequency of occurrence of new HCC lesions remote from the followed-up nodules. The rate was change for 26 months (arrows). d At 32 months, the nodule had enlarged to $23 \mathrm{~mm}$ (arrows). e Histological specimen from rebiopsy showed definite increased cellularity, and diagnosis was well differentiated HCC (bar $50 \mu \mathrm{m})$

$15 \%$ (9 of 60 cases) in LRN cases and $22.7 \%$ (5 of 22 cases) in DN cases. The difference was not statistically significant ( $p=0.4100$, Chi-square test).

The occurrence of new HCC lesions was also reported in our previous study, and was thus confirmed in the present study.

\section{Discussion}

A better knowledge of the natural history of small hepatic nodules including LRN, DN and HCC is important for the 
Table 3 Comparisons of initial profiles in cases with and without malignant transformation of nodules
Data were shown as median and range

a Mann-Whitney test

${ }^{b}$ Fisher's exact test

c Chi-square test

\begin{tabular}{llll}
\hline Parameter & Malignant & Non-malignant & $p$ value \\
\hline Nodule, $n$ & 19 & 73 & - \\
Nodule size $(\mathrm{mm})$ & $12(8-16)$ & $11(8-20)$ & $0.8644^{\mathrm{a}}$ \\
US, hypo/hyper & $13 / 6$ & $54 / 9$ & $0.7727^{\mathrm{b}}$ \\
DN/LRN & $10 / 9$ & $14 / 59$ & $0.0066^{\mathrm{b}}$ \\
Age & $63(24-73)$ & $67(37-79)$ & $0.4719^{\mathrm{a}}$ \\
AST $(\mathrm{IU} / \mathrm{ml})$ & $60(30-197)$ & $73(15-400)$ & $0.1835^{\mathrm{a}}$ \\
ALT $(\mathrm{IU} / \mathrm{ml})$ & $58(11-466)$ & $71(11-466)$ & $0.1835^{\mathrm{a}}$ \\
Platelet $\left(\times 10^{4} / \mu \mathrm{l}\right)$ & $11.7(7.2-20.9)$ & $11.2(3.7-16.7)$ & $0.6477^{\mathrm{a}}$ \\
Child classification/NBNC & $12 / 4 / 3$ & $54 / 11 / 8$ & $0.9680^{\mathrm{c}}$ \\
AFP $(\mathrm{ng} / \mathrm{ml})$ & $12.5(3.1-108.9)$ & $7.9(1.8-657)$ & $0.5505^{\mathrm{a}}$ \\
PIVKA-II $(\mathrm{mAU} / \mathrm{ml})$ & $18(<10-24)$ & $15(<10-31)$ & $0.9382^{\mathrm{a}}$ \\
HCV/HBV/NBNC & $12 / 4 / 3$ & $54 / 11 / 8$ & $0.6469^{\mathrm{c}}$ \\
DM $(+) /(-)$ & $3 / 16$ & $8 / 65$ & $0.6912^{\mathrm{b}}$ \\
Pre-HCC $(+) /(-)$ & $1 / 18$ & $10 / 64$ & $0.2710^{\mathrm{b}}$ \\
\hline
\end{tabular}

However, we were not totally convinced of the result, as the number of LRNs and the lengths of follow-up periods were thought to be insufficient. Therefore, we continued the follow-up study after the previous report was published, and a greater number of LRNs were followed up for longer periods than before. In addition, DNs were followed up for comparison. We also wanted to confirm other results of the previous study, such as the disappearance of lesions in images and the occurrence of new HCCs remote from the target nodules.

The results showed that we could prove the pre-malignant nature of LRNs (Figs. 2 and 3). Although the rate was lower than that of DN, nine of 68 LRNs did show malignant transformation in the present study, quite a different result from our former study [1]. The reason for the difference must simply be attributable to the increases in the number of LRNs and follow-up periods. The difference in the rates of malignant transformation between LRN and $\mathrm{DN}$ is also important, highlighting the merit of classifying non-HCC lesions in cirrhotic liver into LRNs and DNs. We should recognize DNs as high risk lesions and LRNs as low risk lesions.

Except for the pre-malignant nature of LRNs, other phenomena described in our previous report were confirmed in the present study. Disappearance of nodules in images was found in both LRNs and DNs, although the reason for this can still not be conclusively described. However, chronic inflammation and structural change could be involved, with the former perhaps causing new fibrogenesis. The newly-formed fibrous septa would then have separated the nodules into smaller nodules. In any event, the mechanism of disappearance still needs to be clarified.

The occurrence of new HCC lesions remote from target nodules was also confirmed in the present study. As 
discussed previously [1], the new HCC lesion is considered to have developed from de novo-type carcinogenesis, bypassing a rather persistent stage of pre-cancerous nodules. Understanding this phenomenon is a critical issue in daily practical clinical activities [15]. In follow-up examinations, the whole liver should also be carefully examined, as well as these target nodules.

Finally, we hope the findings and considerations of this study will prove useful for practical clinical activities in the management of cirrhotic patients.

Acknowledgements This study (Fukuo Kondo) was supported in part by the grants from The Vehicle Racing Commemorative Foundation.

Compliance with ethical requirements and Conflict of interest Tsunenobu Sato, Fukuo Kondo, Masaaki Ebara, Nobuyuki Sugiura, Shinichiro Okabe, Masahiko Sunaga, Masaharu, Yoshikawa, Eiichiro Suzuki, Sadayuki Ogasawara, Yusuke Shinozaki, Yoshihiko Ooka, Tetsuhiro Chiba, Fumihiko Kanai, Takashi Kishimoto, Yukio Nakatani, Toshio Fukusato, and Osamu Yokosuka declare that they have no conflict of interest. All procedures performed in studies involving human participants were in accordance with the ethical standards of the institutional and/or national research committee and with the 1964 Helsinki declaration and its later amendments or comparable ethical standards. Informed consent was obtained from all individual participants included in the study. This study was approved by an ethics committee of Chiba University School of Medicine.

Open Access This article is distributed under the terms of the Creative Commons Attribution License which permits any use, distribution, and reproduction in any medium, provided the original author(s) and the source are credited.

\section{References}

1. Kondo F, Ebara M, Sugiura N, Wada K, Kita K, Hirooka N, et al. Histological features and clinical course of large regenerative nodules: evaluation of their precancerous potentiality. Hepatology 1990;12:592-598

2. Takayama T, Makuuchi M, Hirohashi S, Sakamoto M, Okazaki $\mathrm{N}$, Takayasu K, et al. Malignant transformation of adenomatous hyperplasia to hepatocellular carcinoma. Lancet 1990;336:1150-1153

3. Rapaccini GL, Pompili M, Caturelli E, Anti M, Aliotta A, Cedrone $\mathrm{A}$, et al. Focal ultrasound lesions in liver cirrhosis diagnosed as regenerating nodules by fine-needle biopsy. Followup of 12 cases. Dig Dis Sci 1990;35:422-427

4. Lencioni R, Caramella D, Bartolozzi C, Di Coscio G. Long-term follow-up study of adenomatous hyperplasia in liver cirrhosis. Ital J Gastroenterol 1994;26:163-168

5. Terasaki S, Kaneko S, Kobayashi K, Nonomura A, Nakanuma Y. Histological features predicting malignant transformation of nonmalignant hepatocellular nodules: a prospective study. Gastroenterology 1998;115:1216-1222

6. Sakamoto M, Hirohashi S. Natural history and prognosis of adenomatous hyperplasia and early hepatocellular carcinoma: multi-institutional analysis of 53 nodules followed up for more than 6 months and 141 patients with single early hepatocellular carcinoma treated by surgical resection or percutaneous ethanol injection. Jpn J Clin Oncol 1998;28:604-608

7. Seki S, Sakaguchi H, Kitada T, Tamori A, Takeda T, Kawada N, et al. Outcomes of dysplastic nodules in human cirrhotic liver: a clinicopathological study. Clin Cancer Res 2000;6:3469-3473

8. Borzio M, Fargion S, Borzio F, Fracanzani AL, Croce AM, Stroffolini T, et al. Impact of large regenerative, low grade and high grade dysplastic nodules in hepatocellular carcinoma development. J Hepatol 2003;39:208-214

9. Kobayashi M, Ikeda H, Hosaka T, Sezaki H, Someya T, Akuta N, et al. Dysplastic nodules frequently develop into hepatocellular carcinoma in patients with chronic viral hepatitis and cirrhosis. Cancer 2006;106:636-647

10. International Working Party. Terminology of nodular hepatocellular lesions. Hepatology 1995;22:983-993

11. International Consensus Group for Hepatocellular Neoplasia. Pathologic diagnosis of early hepatocellular carcinoma: a report of the international consensus group for hepatocellular neoplasia. Hepatology 2009;49:658-664

12. Ebara M, Ohto M, Shinagawa T, Sugiura N, Kimura K, Matsutani $\mathrm{S}$, et al. Natural history of minute hepatocellular carcinoma smaller than three centimeters complicating cirrhosis. A study in 22 patients. Gastroenterology 1986;90:289-298

13. Liver Cancer Study Group of Japan. The General Rules for the Clinical and Pathological Study of Primary Liver Cancer. Third English Edition. Tokyo: Kanehara; 2010

14. Theise ND, Curado MP, Franceschi S, Hytiroglou P, Kudo M, Park YN, et al. Hepatocellular carcinoma. In: Bosman FT, Carneiro F, Hruban RH, Theise ND, editors. World Health Organization Classification of Tumors. WHO Classification of Tumors of the Digestive System. Lyon: International Agency for Research on Cancer; 2010. 205-216

15. Kondo F. Histological features of early hepatocellular carcinomas and their developmental process: for daily practical clinical application. Hepatol Int 2009;3:283-293 